deutend um diesen Punkt, der wohl mit der Temperatur der meist in Frage kommenden Räumlichkeiten nahe genug übereinstimmen wird, so dass eine Correction in dieser Hinsicht überflüssig sein wird. Der Barometerstand schwankte nur wenig um $730 \mathrm{~mm}$. Hier werden freilich sowohl nach der geographischen Lage des Beobachtungsortes als nach den Witterungsverhältnissen grössere Abweichungen eintreten, die man für genauere Bestimmungen leicht berücksichtigen kann, die aber für die praktische Beurtheilung der Frage, ob in einem gegebenen Falle die Luft eines Raumes als mehr oder weniger verunreinigt erklärt werden soll, wohl stets unwesentlich sein werden.

Tabelle zur Vergleichung der Ergebnisse der minimetrischen Methode von Lunge und Zeckendorf mit den Procentgehalt der Luft an Kohlensäure nach Pettenk of er's Methode.

$\begin{array}{cccc}\begin{array}{c}\text { Zahl der Fiillun- } \\ \text { gen der } \\ \text { Kautschukbirne }\end{array} & \begin{array}{c}\text { Procent } \\ \mathrm{CO}_{2}\end{array} & \begin{array}{c}\text { Zahl der Fillum- } \\ \text { gen der } \\ \text { Kautsehukbirne }\end{array} & \begin{array}{c}\text { Procent } \\ \text { CO }_{2}\end{array} \\ 2 & 0,30 & 15 & 0,074 \\ 3 & 0,25 & 16 & 0,071 \\ 4 & 0,21 & 17 & 0,069 \\ 5 & 0,18 & 18 & 0,066 \\ 6 & 0,155 & 19 & 0,064 \\ 7 & 0,135 & 20 & 0,062 \\ 8 & 0,115 & 22 & 0,058 \\ 9 & 0,100 & 24 & 0,054 \\ 10 & 0,09 & 26 & 0,151 \\ 11 & 0,087 & 28 & 0,049 \\ 12 & 0,083 & 30 & 0,048 \\ 13 & 0,08 & 35 & 0,042 \\ 14 & 0,077 & 40 & 0,038 \\ & & 48 & 0,030\end{array}$

Schliesslich möge nicht unerwähnt bleiben, dass wir auch die Anwendung alkoholischer Lösungen in Betracht gezogen haben. Bekanntlich entfärben sich mit Phenolphtalein gefärbte alkoholische Lösungen von Alkalien sebr schnell. H. N. und C. Draper haben dieses Verhalten genau untersucht (Chem. N. 55 S. 143) und schreiben es der Einwirkung atmosphärischer Kohlensäure zu, welche darum so schnell wirkt, weil sie erstens in Alkohol viel leichter als in Wasser löslich ist, und weil zweitens die kohlensauren Alkalien in Alkohol unlöslich sind, also die Entfärbung eintritt, lange ehe sie in Bicarbonate übergeführt sind. Unsere Versuche haben jedoch gezeigt, dass diese Reaction eben wegen ihrer zu grossen Empfindljchkeit unbrauchbar für unseren Zweck ist. Alkohol stundenlang gekocht und bei Abschluss von aller Kohlensäure erkaltet, entfärbt dennoch die Zehntelnormallösung sofort vollständig. Entweder hält er also doch noch zu viel Kohlensäure zurück, oder die Entfärbung hat noch einen anderen als den von den Drapers angenommenen Grund.

\section{Zur Titelfrage für die technischen Chemiker.}

Von

\section{G. Lunge.}

$\mathrm{Zu}$ verschiedenen Malen habe ich die Forderung aufgestellt, dass demjenigen, welcher an einer technischen Hochschule die Staats- oder Diplomprüfung in dem ron mir gewünschten Umfange (vgl. S. 344 d. Z.) bestanden hat, der Titel "Doctor" zustehen solle, und der allgemeine Beifall, mit welchem diese Forderung bei der Hauptversammlung der Gesellschaft für angewandte Chemie am 22. Mai d. J. begrüsst worden ist, sowie eine grössere Anzahl von Briefen hervorragender Docenten an technischen Hochschulen hat mir bewiesen, dass ich einem weit verbreiteten Gefühl Worte gegeben habe. Der Herausgeber dieser Zeitschrift hält (S. 370) für den technischen Chemiker den Titel "Ingenieur" für noch zutreffender, ja ehrenvoller als den des "Doctors", vorausgesetzt natürlich, dass der "Ingenieur" gesetzlich geschützt werde, d. h. Niemand diesen Ehrentitel führen dürfe, der nicht eine entsprechende Prüfung an einer technischen Hochschule bestanden habe.

Ich halte nun dafür, dass beide Vorschläge einander gar nicht ausschliessen ${ }^{1}$ ); auch ich würde es gern begrüssen, wenn der technische Chemiker etwa als „chemischer Ingenieur" bezeichnet würde, entsprechend dem „ingénieur chimiste" oder " "chemical engineer", und habe ja etwas ähnliches in der "Chemischen Industrie“", S. 122 d. J., selbst vorgeschlagen. Aber m. E. genügt dies nicht für den vorliegenden Fall. Erstens halte ich es für kaum möglich und für noch weniger wahrscheinlich, dass im ganzen deutschen Reichsgebiete (und um die Sache wirksam zu machen, müsste in der That noch Österreich und die Schweiz dazu kommen), wo bis jetzt auch die Studirenden der Gewerbeschulen, Techniken und anderer Nicht-Hochschulen nach ihrem Abgange den Titel „Ingenieur" geführt haben, wo übrigens meines Wissens jeder Mensch sich so nennen kann, dem es beliebt, nun mit einem Male der Gebrauch dieses Titels für Jeden straffällig gemacht werden sollte, der nicht an einer technischen $\mathrm{Hochschule}$ studirt und dort eine Diplomprüfung bestanden hat. Dass es mit dem blossen Worte "Ingenieur" durchaus nicht abgethan ist, zeigt u. A. das Beispiel in England, wo eben auch Jeder sich so nennt, der will, wo aber

1) Gewiss nicht. F. 
die tüchtigeren wirklichen Ingenieure ihrem Namen stets den durch Prüfung und Wahl erworbenen Titel: „M. Inst. C. E.“, oder „Assoc. M. Inst. C. E.", d. h. wirkliches oder ausserordentliches Mitglied der Corporation der Civil-Ingenieure nachsetzen, genau ebenso wie die Chemiker das „F. C. S." und „F. I. C." Bei den Engländern wie auch bei den Franzosen werden ja eben sehr wenige Titel vor dem Namen geführt, während es im Deutschen eben einmal anders ist.

Zweitens möchte ich einwenden, dass das Wort "Ingenieur" seinem ganzen bei uns allgemein eingeführten Sinne nach doch gar zu viel Nachdruck auf die mechanische Seite des Berufs legt, um auch für den Chemiker passend zu sein. Die Herleitung des Wortes ist ja nicht die von ingenium in der allgemeinen Bedeutung $=$ Geist, denn das wäre doch ein lächerlicher und noch von Niemandem erhobener Anspruch, dass die Ingenieure die ,geistreichen" Leute par excellence, gegenüber allen anderen Gebieten des Wissens und Schaffens, seien. Vielmehr hat das Wort "ingenium" vermuthlich schon im Mittelalter auch die specielle Bedeutung "Maschine" angenommen, und unbedingt nur daher stammt das Wort "Ingenieur". Im Französischen ist dann die Bedeutung von „engin" noch weiter zusammengeschrumpft, und wird dieses Wort heut meist nur für Hebezeug, Göpel u. dgl. angewendet, doch spricht man auch von "engins de guerre“ im allgemeinen. In der anderen Form "génie" ist dann neben den Bedeutungen Geist, Naturgabe u. s. w. bekanntlich auch diejenige von Ingenieurkunde im allgemeinen und Kriegsbaukunst im besonderen entstanden; aber an chemische Zwecke denkt man dabei gar nicht. Noch deutlicher ist dies in anderen Sprachen. Im Englischen bedeutet engine, im Spanischen ingénio eben einfach "Maschine". So viel ist also sicher, dass bei dem rein französischen Worte "Ingenieur", das nicht, wie "Doctor", der neutralen früheren Weltsprache entstammt und nicht schon durch ein Jahrtausend in Deutschland eingebürgert ist, heutzutage bei uns Niemand an etwas Anderes als an einen Mann denkt, der so gut wie ausschliesslich mit mechanischen oder doch rein physikalischen Mitteln schafft. So sehr ich aber auch bestrebt bin, den technischen Chemiker in der mechanischen Richtung mehr auszubilden (und Niemand wird mir vorwerfen können, dass meine Äusserungen in dieser Hinsicht nicht deutlich genug seien), so geht doch mein stärkster Wunsch dahin, dass der technische Chemiker in erster Linie Chemiker bleibe, dass er die Chemie und nicht das Maschi- nenwesen, als den eigentlichen Grund und Boden seines Schaffens ansehe und sein ganzes Leben lang im lebendigsten Contact mit der reinen Wissenschaft verharre. Lässt sich leugnen, dass dies durch den Titel "Doctor" nicht ungemein richtiger als durch "Ingenieur" ausgedrückt wird? Wie oft kommt es in unserem Fache vor, dass Übertritte aus der Theorie in die Praxis und auch umgekehrt geschehen, während solche Fälle zwischen Physikern und Ingenieuren doch nur ganz selten sind. Dass der technische Chemiker stets in unmittelbarer Beziehung zu einer grundlegenden Naturwissenschaft bleibt, das sollte auch schon durch seinen Titel ausgedrückt sein.

Mein dritter Grund, für den absolvirten technischen Chemiker den Doctortitel zu beanspruchen, ist der, dass jener, im völligen Gegensatze zu den Civil- und Maschineningenieuren, im Kampfe des Lebens die berufliche und sociale Mitbewerbung mit solchen Fachgenossen aufnehmen muss, welche auf Universitäten vorgebildet sind, und dort, ohne dass grössere Intensität ihrer Vorbildung und ihres Hochschulstudiums, oder grössere Wissenschaftlichkeit ihres Studienganges, ihnen ein inneres Recht dazu gäbe, doch einen Titel erwerben, der einmal in der ganzen Welt, vor allem aber doch in Deutschland, als die Signatur eines Mannes, der den höchsten Grad der Bildung erreicht hat, gilt. Mag auch der einsichtige Fabrikant in der Mehrzahl der Fälle (früher war es übrigens entschieden nicht so!) nur wenig darauf geben, ob der von ihm anzustellende Chemiker seinen "Doctor" gemacht hat oder nicht, so denken doch die jungen Leute selbst ganz anders darüber, wofür ich in meinem Vortrage (S. 345) ganz bestimmte Beweise geliefert habe. Schon die Thatsache, dass die Chemiker in manchen Fabriken, wo eine grössere Anzahl derselben angestellt sind, meist sämmtlich "Herr Doctor" angeredet werden, ob sie hierzu ein Recht haben oder nicht, spricht für meine Ansicht. Es ist doch absolut nicht zu leugnen, dass unter mehreren jungen Leuten, welche einander sonst coordinirt sind, diejenigen sich etwas besseres dünken, und mindestens in socialen Beziehungen auch wirklich einen Vorzug geniessen, welche den Doctortitel führen; daher glaubt man höflicherweise auch die dazu nicht Berechtigten so anreden zu müssen, was diesen doch jedesmal einen Stich in's Herz geben muss. So lange man nicht den utopischen Gedanken hegen will, die in allen Ständen und allen Gauen der deutschredenden Länder, die republikanische Schweiz mit eingeschlossen, einmal unbedingt herr- 
schende Titelsucht mit Stumpf und Stiel auszurotten, so lange wird man es nicht verhindern können, dass unter den Chemikern die promovirten Doctoren von der Welt im allgemeinen und in ihren eigenen Kreisen als ein bevorzugter Stand angesehen werden. Gerade weil es eine schreiende Ungerechtigkeit ist, dass die von den Universitäten kommenden Leute sich dieses Vorzuges gegenüber ihren polytechnischen Fachgenossen erfreuen, welche ihnen wissenschaftlich vollkommen gleichstehen und noch eine Menge anderer Sachen haben dazu lernen müssen, gerade darum sollte das Monopol der Universitäten zur Verleihung des Doctortitels, welches gegenüber den ihnen sonst als ebenbürtig anerkannten technischen Hochschulen als ein Anachronismus erscheint, aufgehoben werden, und gerade auch darum, weil in recht vielen Universitätskreisen (Lehrern und Studirenden), wenn sie "entre nous" sind, mit höchst überlegenem Lächeln auf die „banausischen" Emporkömmlinge, die nicht die Universitätsperrücke tragen, herabgeschaut wird. Lassen $\mathrm{ja}$ doch viele, wenn nicht die meisten deutschen Universitäten die an den technischen Hochschulen verbrachten Semester für die Zulassung zur Doctorprüfung gar nicht gelten! Die beste, die einzig würdige Antwort hierauf wäre die Verleibung der Promotionsbefugniss an die technischen Hochschulen. Dann wird man sehen, ob deren Doctoren weniger wissen und weniger leisten als diejenigen der Universitäten. Gleichen Wind und gleiche Sonne für alle, weiter nichts fordern wir.

\section{Zur Asboth'schen Stärkebestimmung.} Von

\section{Dr. C. Monheim.}

Prof. A. v. Asboth behauptet (Chemzg. 1888 No. 42), meine S. 65 d. Z. veröffentlichten Analysen seien nicht genau nach seiner Vorschrift ausgeführt, weil ich nicht immer 0,3 Normalbaryt verwendet habe. Wie aus S. 66 Sp. 2 hervorgeht, ist dieser Einwand völlig hinfällig.

Zu seinen neueren Versuchen hat v. Asboth gereinigte Maisstärke verwendet und damit ziemlich gute Resultate erhalten. $\mathrm{Ob}$ bei Getreidearten auch solche Resultate zu erreichen sind, sollen spätere Versuche zeigen. Mit diesen Ausführungen sind die früheren Angaben v. Asboth's, nach denen besonders die Getreidearten, nach diesem Verfahren untersucht, angeblich gute Resultate gegeben hatten, ganz unvereinbar ${ }^{1}$ ).

Cöln, Juni 1888.

\section{Die Denaturirung des Spiritus.}

Die Unsicherheit, welche über die Denaturirung des Spiritus (sog. Branntweins) in letzter Zeit herrschte (vgl. S. 27 u. 137 d. Z.) ist durch folgenden Bundesrathsbeschluss beseitigt.

Der Bundesrath hat in betreff der Denaturirung des Branntweins in seiner heutigen Sitzung beschlossen:

1. Die durch Beschluss des Bundesraths vom 15. Dezember v. J. (Central-Blatt von 1887, S. 570) vorgeschriebene Zusammensetzung des allgemeinen Denaturirungsmittels im Sinne des Regulativs, betreffend die Steuerfreiheit des Branntweins zu gewerblichen u. dgl. Zwecken, bleibt bis auf Weiteres in Geltung. (S. 27 d. Z.; d. Red.)

2. An die Stelle der bisherigen Bestimmungen über die Beschaffenheit der Bestandtheile des allgemeinen Denaturirungsmittels (Anlage R. 2 des Regulativs) treten die in der Anlage A. enthaltenen Vorschriften. Bis zum 31. Dezember 1888 können jedoch Holzgeist und Pyridinbasen in der den bisherigen Erfordernissen entsprechenden Beschaffenheit zur Denaturirung verwendet werden.

3. Die Prüfung der vorschriftsmässigen Beschaffenheit des Holzgeistes und der Pyridinbasen erfolgt nach Maassgabe der Anleitung in Anlage B.

4. Dem allgemeinen Denaturirungsmittel darf von den zur Zusammensetzung desselben ermächtigten Fabriken ein Zusatz von $40 \mathrm{~g}$ Lavendelöl oder $60 \mathrm{~g}$ Rosmarinöl, auf je $1 l$ beigemengt werden. Die bezüglich der Bestandtheile des allgemeinen Denaturirungsmittels vorgeschriebene Prüfung durch den amtlich bestellten Chemiker ist auf diese $\mathrm{Zu}$ sätze gleichfalls zu erstrecken.

5. Es ist verboten

a) aus denaturirtem Branntwein das Denaturirungsmittel ganz oder theilweise wieder auszuscheiden, oder - abgesehen von der Ausnahme zu 4 - dem denaturirten Branntwein Stoffe beizufügen, durch welche die Wirkung des Denaturirungsmittels in Bezug auf Geschmack oder Geruch verändert wird.

b) Branntwein, welcher - abgesehen von der Ausnahme zu 4 - in der unter a angegebenen Weise behandelt ist, zu verkaufen oder feilzuhalten. Händler mit denaturirtem Branntwein sind verpflichtet, einen Abdruck des vorstehenden Verbots in ihren Verkaufslokalen an einer deutlich sichtbaren Stelle auszuhängen.

6. Gewerbtreibenden kann es gestattet werden, die Denaturirung von Branntwein für den eigenen gewerblichen Bedarf statt mit dem allgemeinen Denaturirungsmittel oder mit Pyridinbasen ( $\$ 10$ des Regulativs) auch mit 5 Proc. Holzgeist von der vorgeschriebenen Beschaffenheit vorzunehmen. Be-

1) Vgl. S. 232 d. Z. D. Red. 